\title{
A Technique for Analyzing the Structure of Atmospheric Turbulence
}

T. C. Kerrigan

September 1978

Prepared for the U.S. Department of Energy under Contract No. EY-76-C-06-1830

Pacific Northwest Laboratory Operated for the U.S. Department of Energy by 


\title{
NOTICE
}

This report was prepared as an account of work sponsored by the United States Government. Neither the United States nor the Department of Energy, nor any of their employees, nor any of their contractors, subcontractors, or their employees, makes any warranty, express or implied, or assumes any legal liability or responsibility for the accuracy, completeness or usefulness of any information, apparatus, product or process disclosed, or represents that its use would not infringe privately owned rights.

The views, opinions and conclusions contained in this report are those of the contractor and do not necessarily represent those of the United States Government or the United States Department of Energy.

\author{
PACIFIC NORTHWEST LABORATORY \\ operated by \\ BATTELLE \\ for the \\ UNITED STATES DEPARTMENT OF ENERGY \\ Under Contract EY-76-C-06-1830
}

Printed in the United States of Am
Available from
National Technical Information Se
United States Department of Comm
5285 Port Royal Road
Springfield, Virginia 22151
Price: Printed Copy S


PNL-2509

$\mathrm{UC}-60$

\section{6}

\section{A TECHNIQUE FOR ANALYZING THE STRUCTURE OF ATMOSPHERIC TURBULENCE}

T. C. Kerrigan

September 1978

Prepared for

the U.S. Department of Energy under Contract EY-76-C-06-1830

Pacific Northwest Laboratory

Richland, Washington 99352 


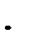




\section{SUMMARY}

The object of this paper is to devise a technique to assess the contribution of large scale coherent gust structures to the statistical properties of atmospheric turbulence. First, an orthonormal family of functions is constructed in terms of which the history of sample paths for a stationary wind process can be analyzed. Next, expressing the state of the wind in terms of the expansion coefficients makes it possible to approximate the wind process by a Markov process. A technique is then developed for estimating the Markov transition probability based on actual wind data. Finally, the form of this transition probability represents an analysis of the gust structure implicit in the wind data. 
- 


\section{CONTENTS}

ABSTRACT . . . . . . . . . . . . . . . . . . . . . .

ACKNOWLEDGMENTS . . . . . . . . . . . . . . . . . . . . iv

I. INTRODUCTION. • • . . . . . . . . . . . . . . . . . . . 1

II. A MARKOV MODEL FOR THE WIND . . . . . . . . . . . . . 3

III. CONCLUDING REMARKS. . . . . . . . . . . . . . . . . 12

REFERENCES . . . . . . . . . . . . . . . . . . . . 15

APPENDIX A: CONSTRUCTION OF A SPECIAL ORTHONORMAL FAMILY . . A-I

APPENDIX B: DERIVATION OF A SPECIAL RECURSION RELATION . . . B-I

APPENDIX C: A BEST ESTIMATE FOR THE PARAMETERS IN A

GAUSSIAN PROBABILITY DENSITY . . . . . . . . . C C-I

APPENDIX D: CONSTRUCTION OF A MULTIPLY GAUSSIAN

PROBABILITY DENSITY. . . . . . . . . . . . . D-1

APPENDIX E: A TECHNIQUE FOR CHOOSING THE VALUE OF A SCALING PARAMETER . . . . . . . . . . . . . E-I

APPENDIX F: AN UPPER BOUND ON A SPECIAL CLASS OF CROSSCOVARIANCE FUNCTIONS. . . . . . . . . . F-1 
Many thanks to J. V. Ramsdell for his encouragement and support of this research. 
I. INTRODUCTION

Interest has recently been expressed in the contribution of large scale coherent gust structures to the statistical properties of atmospheric turbulence [1]. These gust structures may be viewed for example as parcels of vorticity generated by the interaction of the wind with irregular terrain features or as thermals released during conditions of atmospheric instability. once created, these structures evolve and decay in characteristic ways as they are advected downstream. Thus, we hypothesize that atmospheric turbulence is a combination of two kinds of random contributions, these coherent parcels and statistical excursions in the wind velocity which may be viewed as a superposition of a large number of independent random fluctuations. Although this brief description is incomplete at best, the gust hypothesis is sufficiently well-developed for constructive mathematical analysis.

specifically, we envision the following situation. We record the wind velocity at a particular site over a period of time. We assume that the data record is long enough to contain a representative wind sample yet short enough to insure statistical stationarity. The object of this paper is to devise a mathematical technique to determine whether the turbulent fluctuations in the wind record may be viewed as random excursions of a Gaussian process, or whether they are due in part to the passage of characteristic large scale coherent gust structures which make the Gaussian hypothesis untenable [2]. 
Interest in the Gaussian hypothesis arises in part in trying to determine the influence of extreme wind events on wind energy generators [3]. Our analysis provides a wind model which can be used to estimate the irequency of these events even when the Gaussian hypothesis fails. This model provides other benefits as well: a short-term wind prediction which can be used to assess the influence of turbulence on windmill efficiency, a performance evaluation technique which can be used to analyze windmill dynamics. in terms of field data, and conventional input for the Gauss-Markov optimization and control programs in case the Gaussian hypothesis does hold [4]. These applications will not be persued at this time, however.

Finally, we observe the following outline in constructing this turbulence analysis technique. First, we construct an orthonormal family of functions $\left\{\psi_{k}\right\}_{k=1}^{\infty}$ in terms of which we analyze the history of sample paths for the wind process up to a given time $t$. Denoting the wind velocity components by $f_{i}$ for $i=1,2,3$, we can then write

$$
f_{i}(t-s)=\sum_{k=1}^{\infty} \phi_{k i}(t) \psi_{k}(s)
$$

for each $s \geqslant 0$, where $\phi_{k i}$ denotes the $k i \frac{t h}{s}$ expansion coefficient. Second, we derive a recursion of the form

$$
\psi_{n+1}(t+\delta t)=\psi_{n+1}(t)+\sum_{k=1}^{n} c_{n k}(\delta t) \psi_{n}(t)
$$

for $n=0,1,2, \ldots$, where the recursion coefficients $c_{n k}$ are computed 
explicitly. Third, we use this recursion to show that the vector with components $\left\{f_{i}(t), \phi_{k i}(t) \mid i=1,2,3, k=1, \ldots, n\right\}$ may be viewed as a Markov process. Finally, we use the wind record to estimate the transition probability density associated with this Markov process. The structure of this transition probability density represents an implicit analysis of the structure of the turbulence giving rise to the data.

We now proceed to the next section, where we construct this analysis technique. Certain mathematical essentials have been neglected in the belief that the technical demands they pose are beyond the interest of most readers. Omissions will be indicated as they occur, however.

\section{A MARKOV MODEL FOR THE WIND}

We assume that the wind velocity at a fixed point in space may be modeled by an ergodic stationary stochastic process. We simplify our analysis by restricting attention to the horizontal component of wind velocity. Removing this restriction is trivial once the results in this special case have been derived. We assume further that this process is discrete. This assumption allows us to avoid a great deal of mathematical difficulty without seriously diminishing our ability to test the Gaussian hypothesis. Thus, each sample path consists of a sequence of two-dimensional vectors $\left\{\left(f_{1}(i), f_{2}(i)\right) \mid i \in Z\right\}$, where $i$ represents time, $z \equiv\{0, \pm 1, \pm 2, \ldots\}$, and $f_{1}$ and $f_{2}$ denote the coordinates of 
the horizontal wind component in any convenient coordinate system. Let $x \equiv\left\{\left(f_{1}(i), f_{2}(i)\right) \mid i \varepsilon Z\right\}$, where we regard the elements in this case as vector valued random variables, let $\Omega$ denote the set on which the elements of $x$ Iive, let 3 denote the o-algebra of subsets of $\Omega$ generated by the elements of $X$, and let $P$ denote the probability measure on $B$ from which the statistics for the family $\mathrm{X}$ derive. We refer to the pair $(\mathrm{X}, \mathrm{P})$ as our stochastic wind model [2].

The first step in this analysis is to represent the history of the general sample path in terms of a special family of orthonormal functions. We assume that

$$
\sum_{i=0}^{\infty} f_{k}^{2}(j-i) q^{i}<\infty
$$

for all $j$ in $Z$, each $q$ in the interval $(0,1)$ and almost every sample path $\left(f_{1}, f_{2}\right)$. We shall specify a particular value for $q$ later in this section. We refer to Appendix A for the construction of the orthonormal family of polynomials $\left\{\psi_{n}\right\}_{n=1}^{\infty}$ on $z^{+} \equiv\{0,1,2, \ldots\}$. The convergence of the above sums implies the convergence of the orthonormal expansion of the history of almost every sample path [7]:

$$
f_{k}(j-i) \sim \sum_{n=1}^{\infty} \phi_{n k}(j) \psi_{n}(i)
$$

for each $i$ in $z^{+}$and $j$ in $z$, where 


$$
\phi_{n k}(j) \equiv \sum_{i=0}^{\infty} f_{k}(j-i) \psi_{n}(i) q^{i} .
$$

Further, we assume that the set $\left\{\psi_{n}\right\}_{n=1}^{\infty}$ is complete relative to the stochastic model in the sense that

$$
f_{k}(j-i)=\sum_{n=1}^{\infty} \phi_{n k}(j) \psi_{n}(i)
$$

for each $i$ in $Z^{+}, j$ in $Z$, and almost every sample path $\left(f_{1}, f_{2}\right)$. Now, define the vector valued function $\mathrm{U}$ on $\mathrm{Z}$ by

$$
U(j) \equiv\left(f_{1}(j), f_{2}(j), \phi_{11}(j), \phi_{12}(j), \phi_{21}(j), \ldots\right) .
$$

Clearly, specifying $U(j)$ is equivalent to specifying the history of the sample path $\left(f_{1}, f_{2}\right)$ up to time $j$. This is just the representation we seek.

The second step in this analysis is to construct a Markov approximation of the stochastic wind model. First, let E denote expectation with respect to $P$, and define the components of the mean wind vector $\left(\overline{\mathrm{f}}_{1}, \overline{\mathrm{f}}_{2}\right)$ by

$$
\bar{f}_{i} \equiv E f_{i}(\ell)
$$

for $i=1,2$, and the entries in the covariance matrix $\left(\rho_{i j}\right)$ by

$$
\rho_{i j}(k) \equiv E\left(f_{i}(k+l)-\bar{E}_{i}\right)\left(f_{j}(k)-\bar{f}_{j}\right)
$$


for $i, j=1,2$. Stationarity guarantees that $\vec{F}_{i}$ and $\rho_{i j}$ are independent of $\ell$. Define the operator $\Delta$ on the set of real valued functions on $\mathrm{z}$ by

$$
\Delta f(j) \equiv f(j+1)-f(j)
$$

We assume sufficient corditions on the rate at which $\max _{i, j}\left|\rho_{i j}(k)\right| \rightarrow 0$ as $|k| \rightarrow \infty$ so that $\Delta f_{i}$ and $\phi_{n j}$ are essentially independent random variables for $i, j=1,2$ and all $n$ larger than some $N$. Although independent is a stronger qualifier than uncorrelated, we shall assume that uncorrelated random variables are essentially independent. Instead of specifying a value for $\mathrm{N}$ at this point, we merely indicate why such an $N$ should exist. Each $\phi_{n k}(j)$ represents an item of information from the history of the sample path up until time $j$. Call the set $S_{\ell}(j) \equiv\left\{m_{\varepsilon} Z \mid j-\ell \leq m \leq j\right\}$ the recent past relative to $j$. For each $\ell \geqslant 0$ and each sample path $\left(f_{1}, f_{2}\right)$, there exists an $M$ such that the tails

$$
\sum_{n=M+1}^{\infty} \phi_{n k}(j) \psi_{n}(i)
$$

make a negligible contribution to the representation of the sample path in the recent past. By insuring that $\max _{i, j}\left|\rho_{i j}(k)\right| \rightarrow 0$ rapidly as $|k| \rightarrow \infty$, we guarantee the existence of an $\ell^{\prime}$ such that the statistical dependence of $\Delta f_{k}(j)$ on the sample paths before $j-\ell$ ' is negligible. Finally, there exists an $\mathrm{N}$ such that all but a negligible number of the sample paths have the property that 
$M\left(\ell^{\prime}\right) \leq N$. Thus, in a stochastic sense, all but a negligible amount of the information which is helpful in determining the conditional distribution of $\Delta f_{k}(j)$ is represented in the sums

$$
\sum_{n=1}^{N} \phi_{n k}(j) \psi_{n}(i)
$$

Therefore, $\Delta f_{k}(j)$ is essentially independent of $\phi_{n l}(j)$ for $n>N$.

Next, let

$$
x_{n} \equiv\left\{v_{n}(i) \equiv\left(v_{1}(i), \ldots, v_{2 n+2}(i)\right) \mid i \varepsilon z\right\}
$$

denote a set of vector valued random variables defined on a set $\Omega_{n}$. Let $B_{n}$ denote the $\sigma$-algebra of subsets of $\Omega_{n}$ generated by the elements of $x_{n}$ and let $P_{n}$ denote the unique Markov probability measure on $B_{n}$ defined by taking the conditional probability distribution for the random variable $v_{n}(j+1)$ given $v_{n}(j)$ equal to the conditional probability distribution for $U_{n}(j+1)$ given $U_{n}(j)$, where

$$
U_{n}(j) \equiv\left(f_{1}(j), f_{2}(j), \phi_{11}(j), \ldots, \phi_{n 2}(j)\right)
$$

We refer to the pair $\left(\mathrm{x}_{\mathrm{n}}, \mathrm{P}_{\mathrm{n}}\right)$ as the $\mathrm{n}$ th order Markov process derived from $(X, P)$.

Let $\pi_{n}$ denote the projection of a $(2 n+2)$-dimensional vector onto its first two coordinates ard define $\pi_{n} x_{n} \equiv\left\{\pi_{n} V_{n}(i) \mid i \varepsilon z\right\}$. 
We claim that $\left(X_{n}, P_{n}\right)$ is a Markov approximation to $(X, P)$ in the sense that the joint probability distributions for $\left(I_{n} X_{n}, P_{n}\right)$ converge to the joint probability distributions for $(X, P)$.

Rather than furnish a proof of this assertion, we merely indicate why such convergence should occur. As we have already seen, it is possible to find an $N$ such that $\phi_{n i}(k)$ and $\Delta f_{j}(k)$ are essentially independent for $i, j=1,2$ and $n>N$. Thus, the conditional probability distribution for $f_{j}(k+l)$ given $U(k)$ is essentially independent of the variables $\phi_{n i}(k)$ for $i=1,2$ and $n>N$. By virtue of the recursion for $\phi_{n i}(k+1)$ in terms of $f_{i}(k+1), \phi_{1 i}(k)$, $\ldots, \phi_{n i}(k)$ derived in Appendix $B$, we have that the conditional probability distribution for $U_{n}(k+l)$ given $U(k)$ is essentially independent of the variables $\phi_{n i}(k)$ for $i=1,2$ and $n>N$. Equivalently, the conditional probability distribution for $\mathrm{U}_{\mathrm{n}}(\mathrm{k}+1)$ given $\mathrm{U}_{\mathrm{n}}(\mathrm{k})$ is essentially equal to the conditional probability distribution for $U_{n}(k+1)$ given $\left\{U_{n}(i) \mid i \leq k\right\}$. This last statement is just the Markov approximation asserted above.

The final step in this analysis is to approximate the Markov transition probability density for $\left(\mathrm{X}_{\mathrm{n}}, \mathrm{P}_{\mathrm{n}}\right)$ using actual field data. We first take a continuous record of the vector ( $f_{1}(t)$, $\left.f_{2}(t)\right)$ over a period of time which is long enough to contain a representative wind sample yet short enough to insure statistical stationarity. Determining whether the wind record is long enough is dealt with implicitly in Appendix D. To insure stationarity, we assume that the physical conditions responsible for the 
turbulent character of the wind remain constant over the period of time the data are taken. These conditions are primarily atmospheric stability, mean wind velocity and the physical state of the upwind terrain.

We next discretize the wind record. This can be done in a variety of ways. We need only bear in mind that the interpretation of the final result depends on the particular way. Suppose for definiteness that we fix some reference time $t_{0}$ and a time interval $\Delta t$ and agree that $f_{1}(i)$ and $f_{2}(i)$ be the averages of $f_{1}(t)$ and $f_{2}(t)$ for $t$ between $t_{0}+\left(i-\frac{1}{2}\right) \Delta t$ and $t_{0}+\left(i+\frac{1}{2}\right) \Delta t$. $\Delta t$ of course is the scale of resolution required in the analysis.

We next deal with several difficulties which arise in trying to construct the vector $U_{N}(j)$ from a limited number of data of the form $\left(f_{1}(i), f_{2}(i)\right)$, where $i \leq j$. The first difficulty is choosing $q$. Results in Appendix E suggest that $q$ acts like a kind of scaling parameter. In effect, the larger the value of $q$, the greater the emphasis placed on the remote past in extracting information which is useful in constructing the conditional probability distribution for $U_{n}(k+1)$ given $U_{n}(k)$. By virtue of the assumption that $\left\{\psi_{n}\right\}_{n=1}^{\infty}$ forms a complete orthonormal family with respect to expansion of sample path histories, the choice of $\mathrm{q}$ is important only in its influence on the size of $\mathrm{N}$.

The second difficulty in constructing $U_{N}(j)$ is in fact choosing $N$. Recall that $N$ is the largest value of $n$ such that $\phi_{n i}$ and $\Delta f_{j}$ are not essentially independent for some $i$ and $j$. We have assumed that this is the largest value of $n$ for which 
these random variables are significantly correlated for some i and $j$. Deciding whether these variables are correlated is ultimately at the discretion of the person performing the analysis in any particular case.

In practice, the primary consideration in choosing $\mathrm{N}$ is the availability of computational resources. So instead of developing an abstract criterion for choosing $q$ and then $N$, we recommend first choosing the largest affordable $\mathrm{N}$ and then choosing $q$ to maximize the efficiency of the $\mathrm{N}$ th order Markov approximation to $(X, P)$. Appendix $E$ offers a scheme for standardizing this choice for $q$. Further consideration of this point will not be pursued at this time.

The final difficulty in constructing $U_{N}(j)$ is actually computing the series

$$
\phi_{n k}(j) \equiv \sum_{i=0}^{\infty} f_{k}(j-i) \psi_{n}(i) q^{i}
$$

for $n \leq N$ and $k=1,2$. Since the data record is finite, we consider only those $j$ which are so late in the record that the missing tails make statistically insignificant contributions to the series. Appendix $F$ offers an approach to this estimation problem. Further consideration of this point will not be pursued at this time. Once we have computed the components of $U_{N}(j)$ for such a $j$, then computing these components for larger values of $j$ proceeds conveniently by using the recursions developed in Appendix $C$. 
In fact, it is probably most convenient to assume plausible values for these components at the time corresponding to the beginning of the data record and then to obtain all subsequent values by applying these recursions. Only the vectors corresponding to the smallest values of $j$ should be influenced by these initial values. Discarding the vectors $U_{N}(j)$ for $j$ corresponding to these smallest values, we obtain most of the original data recast in the form $\left\{U_{N}(j) \mid j_{1} \leq j \leq j{ }_{2}\right\}$.

Finally, we approximate the Markov transition probability density for the random variable $\mathrm{U}_{\mathrm{N}}(j+1)$ given $\mathrm{U}_{\mathrm{N}}(j)$ using data of the form $\left\{U_{N}(j) \mid j_{1} \leq j \leq j_{2}\right\}$. We first note that we need only estimate the conditional probability density for $\left(f_{1}(j+1)\right.$, $\left.f_{2}(j+1)\right)$ given $U_{N}(j)$ since the remaining components of $U_{N}(j+1)$ are then completely determined by the recursions developed in Appendix B. Let

$$
W_{N}(j) \equiv\left(f_{1}(j+1), f_{2}(j+1), f_{1}(j), f_{2}(j), \phi_{11}(j), \ldots, \phi_{N 2}(j)\right) .
$$

We next note that the transition probability density for $U_{N}(j+1)$ given $\mathrm{U}_{\mathrm{N}}(j)$ is completely determined by the probability density for the random variable $W_{N}$ since the former is just a conditional probability density derived from the latter. And finally, we estimate the probability density for $W_{N}$ by applying the frequency interpretation of probability density to an independent subset of $\left\{w_{N}(j) \mid j_{1} \leq j \leq j_{2}-1\right\}$. Loosely, independence means that any two consecutive vectors in the subset are separated by sufficient time that the components of the latter have forgotten the influence 
of the components of the former. In practice, estimating this separation in the case of the first four components may be based on the rate at which $\max _{i, j}\left|\rho_{i j}(k)\right| \rightarrow 0$ as $|k| \rightarrow \infty$, and estimating this separation in the case of the remaining components may be based on the results of Appendix F. After extracting an essentially independent subset, we appeal to Appendices $C$ and $D$ for our estimate of the probability density for the random variable $W_{N}$. The result takes the form of a convex combination of multivariate Gaussian probability densities. In an abstract sense, each Gaussian density represents a canonical gust structure. If there is exactly one Gaussian density in this representation, we say that the corresponding wind process is Gaussian in the time variable.

\section{CONCLUDING REIARKS}

The object of this paper has been to devise a technique for estimating the number of Gaussian components in a stochastic process based on a sequence of wind data. In reality, of course, not even isotropic turbulence conforms to the Gaussian hypothesis [10]. Nevertheless, this technique should be useful in estimating the number of turbulent modes spawned under given conditions of geography and meteorology. If this analysis should reveal more than one such mode, then estimates of the frequency of the wind events considered in [3] for example should be reconsidered. Under these conditions, we expect that estimates based on the multiply Gaussian model would be more realistic than those based on the singly Gaussian model. 
As noted at the end of section II, estimating the number of Gaussian components of the probability density for the random variable $W_{N}$ is tantamount to estimating the number of Gaussian components of the transition probability density for the $\left(\mathrm{X}_{\mathrm{N}}, \mathrm{P}_{\mathrm{N}}\right)$ process. We have thus accomplished our objective without actually exhibiting the transition probability density. The wind model implicit in this density is of interest for its own sake, however. In fact, a paper using this wind model to assess the influence of turbulence on the efficiency of wind energy generators should be out shortly.

In addition, the technique we have developed for analyzing the structure of atmospheric turbulence generalizes immediately to the analysis of any stationary vector valued process. For example, it applies to the problem of determining the aerodynamic characteristics of vertical-axis wind energy generators using actual field data. In this case, we take wind speed as the first vector component and torque measured at the base of the rotor shaft as the second. A paper on this application should also be out shortly.

Finally, this Markov analysis may be viewed as a variation on the conventional spectral analysis. The basis for comparison is the similarity between representing sample path histories in terms of $\left\{\psi_{n}\right\}_{n=1}^{\infty}$ and representing entire sample paths in terms of powers of the exponential function. Thus, issues such as stationarity, site specificity, numerical sensitivity and statistical significance have in a general sense been treated in the literature 
already. In fact, a comprehensive treatment of these topics in this setting is clearly beyond the scope of this development. In any case, this analysis technique is sufficiently well-developed that conclusions based on its application should not be reversed by strengthening the theoretical underpinnings. 


\section{REFERENCES}

[1] Liu, J. T. C. and A. Alper: "On the Large-Scale Structure in Turbulent Free Shear Flows," Proceedings of Symposium on Turbulent Shear Flows, April 18-20-1977, The Pennsylvania State University, University Park, Pennsylvania, April 1977, pp. 11.1 - 11.11.

[2] Cramer, Harald and M. R. Leadbetter: Stationary and Related Stochastic Processes. Sample Function Properties and Their Applications. John Wiley \& Sons, Inc., 1967, 348 pp.

[3] Kerrigan, T. C.: Spectral Estimates of a Wind Fluctuation Statistic Pertaining to wind Energy Generators. PNL-2511. Battelle, Pacific Northwest Laboratories, Richland, washington, December 1977 .

[4] Meditch, J. S.: Stochastic Optimal Linear Estimation and Control. McGraw- Hill Book Company, 1969, 394 pp.

[5] Rudin, Walter: Principles of Mathematical Analysis. MCGraw-Hill Book Company, 1964, 270 pp.

[6] Nering, Evar D.: Linear Algebra and Matrix Theory. John Wiley and Sons, 1963, $289 \mathrm{pp}$.

[7] Apostol, Tom M.: Mathematical Analysis. A Modern Approach to Advanced Calculus. Addison-Wesley Publishing Co., Inc. 1964, 559 Pp.

[8] Hogg, Robert V. and Allen T. Craig: Introduction to Mathematical Statistics. The Macmillan Co,, New York, NY. 1965. 383 PP.

[9] Doob, J. I.: Stochastic Processes. John Wiley \& Sons, Inc. 1953. $654 \mathrm{pp}$.

[10] Batchelor, G. K.: The Theory of Homogeneous Turbulence. Cambridge at the university press. 1970. $197 \mathrm{pp}$. 

APPENDIX A

CONSTRUCTION OF A SPECIAL ORTHONORMAL FAMILY 


\section{APPENDIX A}

CONSTRUCTION OF A SPECIAL ORTHONORMAL FAMILY

Let $\ell_{2}(q)$ denote the set of real valued functions $f$ on $\mathrm{z}^{+} \equiv\{0,1,2, \ldots\}$ with the property that

$$
\sum_{k=0}^{\infty} f^{2}(k) q^{k}<\infty,
$$

where $q$ denotes some fixed number in the interval $(0,1)$. Define the inner product of any two elements $f$ and $g$ in $\imath_{2}(q)$ by

$$
(f, g) \equiv \sum_{k=0}^{\infty} f(k) g(k) q^{k} .
$$

$\ell_{2}(q)$ becomes a Hilbert space [5] with respect to this inner product. The object of this section is to construct a special orthonormal subset $\left\{\psi_{n}\right\}_{n=1}^{\infty}$ of this Hilbert space.

We obtain $\left\{\psi_{n}\right\}_{n=1}^{\infty}$ by applying the Gram-Schmidt orthonormaiization process [6] to the polynomials $\left\{\zeta_{n}\right\}_{n=1}^{\infty}$, where

$$
\zeta_{n}(k) \equiv k^{n-1}
$$

for $\mathrm{k}$ in $\mathrm{z}^{+}$. We first turn attention to a lemma which will help in computing inner products. Define

$$
S(n) \equiv \sum_{k=0}^{\infty} k^{n} q^{k}
$$

for $n=0,1,2, \ldots$. Then 
Lemma Al $: \quad S(n+1)=\frac{q}{1-q} \sum_{k=0}^{n}\left(\begin{array}{c}n+1 \\ k\end{array}\right) S(k)$.

$$
\text { Pf: } \begin{aligned}
& \sum_{k=0}^{n}\left(\begin{array}{l}
n \\
k
\end{array}\right) s(k) \\
= & \sum_{k=0}^{n}\left(\begin{array}{l}
n \\
k
\end{array}\right) \sum_{j=0}^{\infty} j k_{q}^{j} \\
= & \sum_{j=0}^{\infty}\left(\sum_{k=0}^{n}\left(\begin{array}{l}
n \\
k
\end{array}\right) j^{k}\right) q^{j} \\
= & q^{-1} \sum_{j=0}^{\infty}(j+1) q^{j+1} \\
= & q^{-1} s(n) .
\end{aligned}
$$

Thus;

$$
s(n)=q \sum_{k=0}^{n}\left(\begin{array}{l}
n \\
k
\end{array}\right) s(k) .
$$

Therefore,

$$
s(n)=\frac{q}{1-q} \sum_{k=0}^{n-1}\left(\begin{array}{l}
n \\
k
\end{array}\right) s(k)
$$

In addition,

$$
S(0)=\sum_{k=0}^{\infty} q^{k}=\frac{q}{1-q} .
$$

Thus, the lemma provides the means to compute $s(n)$ for arbitrary $n$. 
Next, define $\|f\| \equiv \sqrt{(f, f)}$ for $f$ in $\ell_{2}(q)$. In order to facilitate the construction of $\left\{\psi_{n}\right\}_{n=1}^{\infty}$, we simultaneously construct an auxiliary family of functions $\left\{^{\prime} \xi_{n^{\prime}}{ }^{\infty}=1\right.$ which satisfies

$$
\xi_{n+1}=\zeta_{n+1}-\sum_{k=1}^{n}\left(\zeta_{n+1}, \psi_{k}\right) \psi_{k},
$$

and

$$
\psi_{n+1}=\xi_{n+1} /\left\|\xi_{n+1}\right\|
$$

These are in fact the Gram-Schmidt recursion relations, and $\xi_{n+1}$ is clearly just a nonnormalized version of $\psi_{n+l}$.

We begin this construction by setting

$$
\psi_{1} \equiv \xi_{1} \equiv \sqrt{1-q}
$$

the normalized version of $\zeta_{I}$. Proceeding by induction, we assume that we have computed $\psi_{n}$ and $\xi_{n}$ in the form

$$
\psi_{n}(j)=\sum_{i=1}^{n} a_{n i} j^{i-1},
$$

and

$$
\xi_{n}(j)=\sum_{i=1}^{n} b_{n i} j^{i-1} .
$$

Our solution to the problem of constructing $\left\{\psi_{n}\right\}_{n=1}^{\infty}$ is to use 
the Gram-Schmidt recursion relations to derive recursion relations for the coefficients of these polynomials:

$$
\begin{aligned}
& \left(\zeta_{n+1} \psi_{k}\right) \\
= & \sum_{j=0}^{\infty} j^{n}\left(\sum_{i=1}^{k} a_{k i} j^{i-1}\right) q^{j} \\
= & \sum_{i=1}^{k} a_{k i} \sum_{j=0}^{\infty} j^{n+i-1} q^{j} \\
= & \sum_{i=1}^{k} a_{k i} s(n+i-1) .
\end{aligned}
$$

\section{Thus,}

$$
b_{n+1, j}=\delta_{n+1}(j)-\sum_{k=1}^{n}\left(\sum_{i=1}^{k} a_{k i} s(n+i-1)\right) a_{k j} \text {. }
$$

Therefore,

$$
b_{n+1, j}=\delta_{n+1}(j)-\sum_{i=1}^{n} s(n+i-1) \sum_{k=i}^{n} a_{k i} a_{k j},
$$

with the understanding that $a_{k j}=0$ in case $k<j$. Next, 


$$
\begin{aligned}
& \left\|\xi_{n+1}\right\|^{2} \\
= & \sum_{k=0}^{\infty}\left(\sum_{i=1}^{n+1} b_{n+1, i} k^{i-1}\right)\left(\sum_{j=1}^{n+1} b_{n+1, j} k^{j-1}\right) q^{k} \\
= & \sum_{i=1}^{n+1} \sum_{j=1}^{n+1} b_{n+1, i} b_{n+1, j} \sum_{k=0}^{\infty} k^{i+j-2} q^{k} .
\end{aligned}
$$

Therefore,

$$
\left\|\xi_{n+1}\right\|^{2}=\sum_{i=1}^{n+1} \sum_{j=1}^{n+1} s(i+j-2) b_{n+1, i} b_{n+1, j} .
$$

Finally,

$$
a_{n+1, j}=b_{n+1, j} /\left\|\xi_{n+1}\right\|
$$

These recursion relations for $s(n), b_{n j},\left\|\xi_{n}\right\|^{2}$ and $a_{n j}$ provide the means to compute each element in the set $\left\{\psi_{n}\right\}_{n=1}^{\infty}$. 

APPENDIX B

DERIVATION OF A SPECIAL RECURSION RELATION 


\section{APPENDIX B}

\section{DERIVATION OF A SPECIAL RECURSION RELATION}

Let $f$ denote a real valued function with the property that

$$
\sum_{i=0}^{\infty} f^{2}(j-i) q^{i}<\infty
$$

for each $j$ in $z \equiv\{0, \pm 1, \pm 2, \ldots\}$, where $q$ denotes some fixed number in the interval $(0,1)$. Define the functions $\left\{\phi_{n}\right\}_{n=1}^{\infty}$ by

$$
\phi_{n}(j) \equiv \sum_{i=0}^{\infty} f(j-i) \psi_{n}(i) q^{i}
$$

for $j$ in $z$, where $\left\{\psi_{n}\right\}_{n=1}^{\infty}$ is the orthonormal subset of $l_{2}(q)$ constructed in Appendix A. The object of this section is to derive a special recursion relation for $\left.\phi_{n}\right\}_{n=1}^{\infty}$ which is inherited from one for $\left\{\psi_{n}\right\}_{n=1}^{\infty}$.

We first derive the recursion relation for $\left\{\psi_{n}\right\}_{n=1}^{\infty}$. Define the operator $\Delta$ on the set of real valued functions on $z$ by

$$
\Delta g(j) \equiv g(j+1)-g(j)
$$

Now since $\psi_{n+1}$ is a polynomial of order $n$, we may write $\Delta \psi_{n+1}$ uniquely as a linear combination of $\psi_{1}, \ldots, \psi_{n}$. In fact,

$$
\Delta \psi_{n+1}=\sum_{k=1}^{n} c_{n k} \psi_{k},
$$


where $c_{n k} \equiv\left(\Delta \psi_{n+l}, \psi_{k}\right)$. To compute this inner product, first note that

$$
\begin{gathered}
\zeta_{2+1}(j) \\
=(j+1)^{l}-j^{2} \\
=\sum_{i=0}^{\ell-1}\left(\begin{array}{l}
l \\
i
\end{array}\right) j^{i} .
\end{gathered}
$$

so,

$$
n_{n+1}(j)=\sum_{\ell=1}^{n+1} a_{n+1, \ell} j^{\ell-1}
$$

inplies that

$$
\Delta \psi_{n+1}(j)=\sum_{l=2}^{n+1} a_{n+1, l} \sum_{i=0}^{\ell-2}\left(\begin{array}{c}
l-1 \\
i
\end{array}\right) j^{i} .
$$

Thus,

$$
\begin{aligned}
& \left(\Delta \psi_{n+1}, \psi_{k}\right) \\
= & \sum_{j=0}^{\infty}\left(\sum_{\ell=2}^{n+1} a_{n+1, i} \sum_{i=0}^{2-2}\left(\begin{array}{c}
\ell-1 \\
i
\end{array}\right) j^{i}\right)\left(\sum_{m=1}^{k} a_{k m} j^{m-1}\right) q^{j} \\
= & \sum_{\ell=2}^{n+1} \sum_{m=1}^{k} a_{n+1, \ell} a_{k m} \sum_{i=0}^{l-2}\left(\begin{array}{c}
\ell-1 \\
i
\end{array}\right) \sum_{j=0}^{\infty} j^{i+m-1} q^{j} \\
= & \sum_{\ell=1}^{n} \sum_{m=1}^{k} a_{n+1, \ell+1} a_{k m} \sum_{i=0}^{i-1}\left(\begin{array}{c}
l \\
i
\end{array}\right) s(i+m-1) .
\end{aligned}
$$


Therefore,

$$
\Delta \psi_{n+1}=\sum_{k=1}^{n} c_{n k} \psi_{k}
$$

where

$$
c_{n k}=\sum_{\ell=1}^{n} \sum_{m=1}^{k} a_{n+1, \ell+1} a_{k m} \sum_{i=0}^{\ell-1}\left(\begin{array}{l}
l \\
i
\end{array}\right) s(i+m-1) .
$$

We next derive the consequences of this recursion relation for $\left\{\phi_{\mathrm{n}}\right\}_{\mathrm{n}=1}^{\infty}$ :

$$
\begin{aligned}
& \Delta \phi_{n+1}(j) \\
= & \sum_{i=-\infty}^{j} f(i) \psi_{n+1}(j-i) q^{j-i} \\
= & \sum_{i=-\infty}^{j+1} f(i) \psi_{n+1}(j+1-i) q^{j+1-i} \\
& -\sum_{i=-\infty}^{j} f(i) \psi_{n+1}(j-i) q^{j-i} \\
& f(j+1) \psi_{n+1}(0) \\
& +q \sum_{i=-\infty}^{j} f(i)\left(\Delta \psi_{n+1}(j-i)\right) q^{j-i} \\
& +\sum_{i=-\infty}^{j} f(i) \psi_{n+1}(j-i) q^{j-i}
\end{aligned}
$$




$$
\begin{aligned}
& \quad-\sum_{i=-\infty}^{j} f(i) \psi_{n+1}(j-i) q^{j-i} \\
& =\psi_{n+1}(0) f(j+1) \\
& +q \sum_{i=-\infty}^{j} f(i) \sum_{k=1}^{n} c_{n k} \psi_{k}(j-i) q^{j-i} \\
& \quad-(1-q) \sum_{i=-\infty}^{j} f(i) \psi_{n+1}(j-i) q^{j-i} \\
& =\psi_{n+1}(0) \pm(j+1) \\
& \quad+q \sum_{k=1}^{n} c_{n k} \phi_{k}(j) \\
& \quad-(1-q) \phi_{n+1}(j) .
\end{aligned}
$$

Therefore,

$$
\phi_{n+1}(j+1)=\psi_{n+1}(0) f(j+1)+q \sum_{k=1}^{n+1} c_{n k} \phi_{k}(j),
$$

for $n=0,1,2, \ldots$, where $c_{n, n+1} \equiv 1$. Verification of this relation for $n=0$ is a special case of the computation given above. 
APPENDIX C

A BEST ESTIMATE FOR THE PARAMETERS

IN A GAUSSIAN PROBABILITY DENSITY 


\section{APPENDIX C}

\section{A BEST ESTIMATE FOR THE PARAMETERS \\ IN A GAUSSIAN PROBABIIITY DENSITY}

Let $E_{n}$ denote $\mathrm{n}$-dimensional Euclidean space and suppose the set $\left\{\mathrm{y}_{\mathrm{k}}\right\}_{\mathrm{k}=1}^{\mathrm{m}} \subseteq \mathrm{E}_{\mathrm{n}}$ represents $\mathrm{m}$ independent samples from a collection of vectors with probability density given by

$$
p(y) \equiv \frac{\sqrt{|P|}}{(2 \pi)^{n / 2}} \exp \left(-\frac{1}{2}(y-\bar{y}) * P(y-\bar{y})\right)
$$

where $\bar{Y} \varepsilon E_{n}$ and $P \equiv\left(p_{i j}\right)$, an $n \times n$ positive definite quadratic form. The object of this section is to compute best estimates for $\bar{y}$ and $P$ in terms of $\left\{y_{k}\right\}_{k=1}^{m}$.

We first state a criterion by which to select these best estimates. Let $x_{1}, \ldots, x_{n}$ denote $m$ independent random variables such that each has probability density $p(x)$. Then the probability density for the vector $\left(x_{1}, \ldots, x_{n}\right)$ is given by the product

$$
\prod_{k=1}^{m} p\left(x_{k}\right)
$$

We define the likelihood $L$ [8] that the data $\left\{y_{j}\right\}_{j=1}^{\mathrm{m}}$ are $\mathrm{m}$ independent samples from a collection with probability density given by $p$ as

$$
L(\bar{y}, P) \equiv \prod_{k=1}^{m} p\left(y_{k}\right)
$$


Our best estimate for the pair $(\bar{y}, P)$ is the one which maximizes L. We devote the remainder of this section to computing this estimate by computing the unique solution to the system

$$
\Gamma_{\bar{Y}} L(\bar{y}, P)=0
$$

and

$$
\frac{\partial}{{ }^{3} P_{i j}} L(\bar{y}, P)=0
$$

for $i, j=1, \ldots, n$.

It will become clear that this estimate for $\bar{y}$ is independent of the choice of $\mathrm{P}$. We proceed by first estimating $\bar{Y}$ and then $P$.

$$
\begin{aligned}
& L(\bar{y}, P) \\
= & \prod_{k=1}^{m} \frac{\sqrt{|P|}}{(2 \pi)^{n / 2}} \exp \left(-\frac{1}{2}\left(y_{k}-\bar{y}\right) * P\left(y_{k}-\bar{y}\right)\right) \\
= & \left(\frac{\sqrt{|P|}}{(2 \pi)^{n / 2}}\right)^{m} \in \exp \left(-\frac{1}{2} \sum_{k=1}^{m}\left(y_{k}-\bar{y}\right) * P\left(y_{k}-\bar{y}\right)\right)
\end{aligned}
$$

implies that 


$$
\begin{aligned}
& \nabla_{\bar{y}} L(\bar{y}, P) \\
= & \left(\frac{\sqrt{|P|}}{(2 \pi)^{n / 2}}\right)^{m} \exp \left(-\frac{1}{2} \sum_{k=1}^{m}\left(y_{k}-\bar{y}\right) * P\left(y_{k}-\bar{y}\right)\right) \\
= & \left(\frac{\sqrt{|P|}}{(2 \pi)^{n / 2}}\right)^{m} \exp \left(-\frac{1}{2} \sum_{k=1}^{m}\left(y_{k}-\bar{y}\right)\right) \\
P & \left(\left(\sum_{k=1}^{m} y_{k}\right)-\frac{\bar{y}}{m} \bar{y}\right)
\end{aligned}
$$

Thus,

$$
\nabla_{\bar{y}} L(\bar{y}, P)=0
$$

implies that

$$
\overline{\mathrm{y}}=\frac{1}{\mathrm{~m}} \sum_{\mathrm{k}=1}^{\mathrm{m}} \mathrm{y}_{\mathrm{k}}
$$

We may facilitate the computation of $\mathrm{P}$ by centering the coordinate system at $\bar{y}$. Adopting this point of view, we have that

$$
L(P)=\left(\frac{\sqrt{|P|}}{(2 \pi)^{n / 2}}\right)^{m} \exp \left(-\frac{1}{2} \sum_{i, j=1}^{n} p_{i j} \sum_{k=1}^{m} y_{k i} y_{k j}\right),
$$

where $y_{k i}$ denotes the $i$ th component of $y_{k}$. Let $P_{i j}$ denote the 
cofactor of the element $p_{i j}$ relative to the matrix $P$, and recall that $p_{i j}=p_{j i}$ for $i, j=1, \ldots, n$ and

$$
|P|=\sum_{j=1}^{n} p_{i j}\left|p_{i j}\right|
$$

for $i=1, \ldots, n$. Thus,

$$
\begin{aligned}
& \frac{1}{2}\left(\frac{\partial}{\partial P_{r s}}+\frac{\partial}{j D_{S r}}\right)\left(\frac{\sqrt{|P|}}{(2 \pi)^{n / 2}}\right)^{m} \\
& =m\left(\frac{\sqrt{|P|}}{(2 \pi)^{n / 2}}\right)^{m-1} \frac{\frac{1}{2(2 \pi)^{n / 2} \sqrt{|P|}}}{2}\left(\frac{\partial}{\partial P_{r s}}+\frac{\partial}{\partial P_{s r}}\right)|P| \\
& =\frac{m}{2(2 \pi)^{n / 2} \sqrt{|P|}}\left(\frac{\sqrt{|P|}}{(2 \pi)^{n / 2}}\right)^{m-1} \frac{1}{2}\left(\left|P_{r s}\right|+\left|P_{s r}\right|\right) \\
& =\frac{m}{2}\left(\frac{|P|}{(2 \pi)^{n / 2}}\right)^{m} \frac{I_{r s} \mid}{|P|},
\end{aligned}
$$

and

$$
\begin{aligned}
& \frac{1}{2}\left(\frac{\partial}{\partial p_{r s}}+\frac{\partial}{\partial p_{s r}}\right) \exp \left(-\frac{1}{2} \sum_{i, j=1}^{n} p_{i j} \sum_{k=1}^{m} y_{k i} y_{k j}\right) \\
= & \left(-\frac{1}{2} \sum_{k=1}^{m} y_{k r} y_{k s}\right) \exp \left(-\frac{1}{2} \sum_{i, j=1}^{n} p_{i j} \sum_{k=1}^{m} y_{k i} y_{k j}\right)
\end{aligned}
$$

imply that 


$$
\begin{gathered}
\frac{1}{2}\left(\frac{\partial}{\partial P_{r S}}+\frac{\partial}{\partial P_{S I}}\right) L(P) \\
=\left(\frac{m}{2} \frac{\left|P_{r S}\right|}{|P|}-\frac{1}{2} \sum_{k=1}^{m} y_{k r} Y_{k s}\right) L(P) .
\end{gathered}
$$

Thus,

$$
\frac{\partial}{\partial P_{i j}} L(P)=0
$$

for $i, j=1, \ldots, n$ implies that the $i j \underline{\text { th }}$ entry in the matrix $p^{-1}$ is given by $\frac{1}{m} \sum_{k=1}^{m} y_{k i} y_{k j}$. Returning to the original coordinate system, we have that

$$
\left(P^{-1}\right)_{i j}=\frac{1}{m} \sum_{k=1}^{m}\left(y_{k i}-\bar{y}_{i}\right)\left(y_{k j}-\bar{y}_{j}\right)
$$


APPENDIX D

CONSTRUCTION OF A MULTIPLY GAUSSIAN PROBABILITY DENSITY 
APPENDIX D

CONSTRUCTION OF A MULTIPLY

GALSSIAN PROBABILITY DENSITY

The object of this section is to develop a technique for assigning a best probability density to a finite subset of $n-$ dimensional Euclidean space $E_{n}$. We regard each element in this subset as an independent representation of the state of the wind as described in section II. Since our intention is to test the gust model proposed in section $I$, we cast this probability density in the form of a convex combination of multivariate Gaussian probability densities. In an abstract sense, each Gaussian component represents a canonical gust structure. Thus, assigning a probability density of this form constitutes an implicit analysis of the structure of atmospheric turbulence.

Our first task is to develop a measure of the likelihood that a finite subset $S \subseteq E_{n}$ represents a collection of independent samples from a Gaussian population. This development may be viewed as an extension of Appendix $c$. Set $S \equiv\left\{y_{j}\right\}_{j=1}^{m}$, and assume for convenience that

$$
\sum_{j=1}^{m} y_{j}=0
$$

and that the linear span of $S$ is $E_{n}$. Define the matrix $P$ by

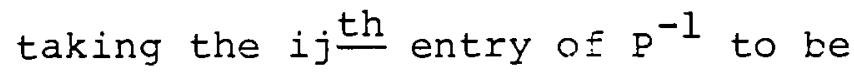

$$
\left(\mathrm{P}^{-1}\right)_{i j} \equiv \frac{1}{\mathrm{~m}} \sum_{k=1}^{\mathrm{m}} \mathrm{y}_{k i} \mathrm{y}_{k j},
$$


where $y_{k i}$ denotes the $i$ th component of $y_{k}$. Let $p$ denote the probability density given by

$$
p(y) \equiv \frac{\sqrt{|P|}}{(2 \pi)^{n / 2}} \exp \left(-\frac{1}{2} y^{\star P y}\right)
$$

and reconsider $L$ as a function of $S$ :

$$
L(S) \equiv \prod_{j=1}^{m} p\left(y_{j}\right)
$$

According to Appendix $C$, our choice of $P$ maximizes $L(S)$. Thus, we regard $L(S)$ as a suitable measure of the likelihood that the elements of $S$ represent independent samples from an unspecified Gaussian population.

In order to extend this notion to a larger class of sets $s$, we first derive a more useful expression for $L(S)$ :

$$
\begin{aligned}
& =\left(\frac{\sqrt{|\mathrm{P}|}}{(2 \pi)^{\mathrm{n} / 2}}\right)^{\mathrm{m}} \exp \left(-\frac{1}{2} \sum_{k=1}^{m} \mathrm{y}_{k}{ }^{\star} \mathrm{P} \mathrm{y}_{k}\right) \\
& =\left(\frac{\sqrt{|\mathrm{P}|}}{(2 \pi)^{\mathrm{n} / 2}}\right)^{\mathrm{m}} \exp \left(-\frac{1}{2} \sum_{i, j=1}^{n} p_{i j} \sum_{k=1}^{m} y_{k i} y_{k j}\right) \\
& =\left(\frac{\sqrt{|P|}}{(2 \pi)^{n / 2}}\right)^{m} \exp \left(-\frac{m}{2} \sum_{i, j=1}^{n} p_{i j}\left(P^{-1}\right) i j\right)
\end{aligned}
$$




$$
\begin{aligned}
& =\left(\frac{\sqrt{|P|}}{(2 \pi)^{n / 2}}\right)^{m} \exp \left(-\frac{m n}{2}\right) \\
& =\left[\prod_{k=1}^{n}\left(\frac{\lambda_{k}}{2 \pi e}\right)^{m / 2}\right],
\end{aligned}
$$

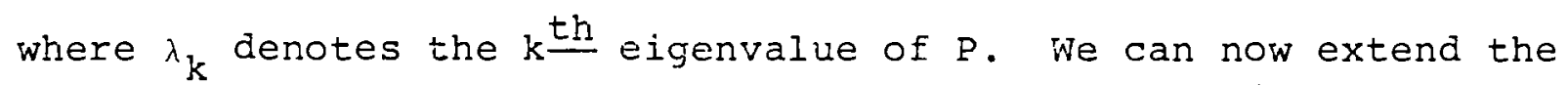
definition of $L$ to the collection of all finite nonempty subsets $S \subseteq E_{n}$ by taking

$$
L(S) \equiv\left[\prod_{k^{\prime} \neq 0}\left(2 \pi e \lambda_{k}\right)\right]^{-\frac{m}{2}},
$$

where the product is taken over the nonzero eigenvalues of the matrix whose ijth entry is

$$
\frac{1}{m} \sum_{k=1}^{m}\left(y_{k i}-\bar{y}_{i}\right)\left(y_{k j}-\bar{y}_{j}\right),
$$

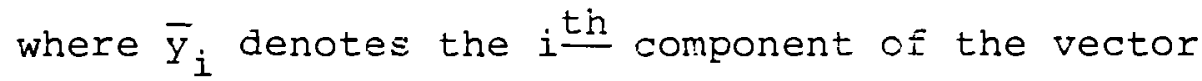

$$
\frac{1}{\mathrm{~m}} \sum_{\mathrm{k}=1}^{\mathrm{m}} \mathrm{y}_{\mathrm{k}}
$$

our second task is to develop a measure of the separation between two finite subsets $S$ and $T$ of $E_{n}$. We base the concept of separation on the gust model proposed in section I. Let o(S) denote the number of elements in the set $S$ ard $S+T$ denote the union of $S$ and $T$ without consolidating duplications. Thus $O(S+T)=O(S)+\circ(\dot{T})$. 
Loosely, $L(S)(d y)^{\circ(S)}$ is the probability that the elements of $S$ denote independent samples from some Gaussian population. If the elements of $\mathrm{S}+\mathrm{T}$ are independent representations of the state of the wind, then $I(S) L(T)(d y)^{O(S+T)}$ is the probability that $S$ is a set of representations from one such population and $\mathrm{T}$ is a set of representations from another. Further, $I(S+T)(d y) \circ(S+T)$ is the probability that the elements of $S+T$ are independent representations from the same population. Thus,

$$
o(S, T)=\frac{L(S) L(T)}{L(S+T)}
$$

is a measure of the separation between $S$ and $T$ in the sense that if $\rho(S, T)>1$, then $S$ and $T$ are more likely to have arisen from different Gaussian populations.

Now let $\left\{\mathrm{X}_{i}\right\}_{i=1}^{\mathrm{k}}$ denote $\mathrm{k}$ independent representations of the state of the wind. Our third task is to partition this set into subsets which correspond to the gust structures proposed in section $I$. We proceed by induction. We start by partitioning $\left\{\mathrm{x}_{\mathrm{i}}\right\}_{\mathrm{i}=1}^{\mathrm{k}}$ into $\mathrm{k}$ subsets $\mathrm{s}_{1} \ldots \mathrm{s}_{\mathrm{k}}$ such that $\mathrm{s}_{\mathrm{i}} \equiv\left\{\mathrm{x}_{\mathrm{i}}\right\}$. The general induction goes as follows. Let $s_{1}, \ldots, s_{l}$ denote the elements in the partition after $\mathrm{k}-l$ steps. Now form

$$
d_{\ell} \equiv \min _{1 \leq i<j \leq \ell} \rho\left(s_{i}, s_{j}\right)
$$

and select $i$ ' and $j '$ such that 


$$
d_{2}=p\left(s_{i}, s_{j}\right)
$$

Of all possible pairs, $S_{i}$, and $S_{j}$, are closest according to the criterion developed above. Now replace $s_{i}$, by $s_{i},+s_{j}$, and $S_{j}$, by $S_{\ell}$. Thus, we nave reduced the order of the partition from $\ell$ to $\ell-1$ in a way which is consistent with the gust model. Finally, we terminate the induction when $\min _{i<j} \rho\left(S_{i}, S_{j}\right)$ is large and when each element of the partition contains enough vectors to support a statistically significant Gaussian probability density. Definitions of the terms "large" and "statistically significant" are presently unspecified. They constitute part of a comprehensive treatment of this subject which awaits further investigation of the mathematical properties of $\rho$.

We conclude this section by associating the probability density p defined by

$$
p(x) \equiv k^{-1} \sum_{j=1}^{2} \circ\left(s_{i}\right) p_{j}(x)
$$

with the data $\left\{x_{i}\right\}_{i=1}^{k}$, where $p_{j}$ is the Gaussian probability

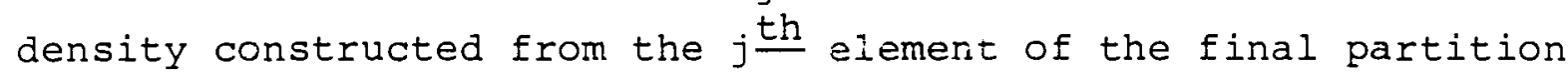
$\left\{s_{j}\right\}_{j=1}^{l}$ using the technique developed in Appendix $c$. If $\ell>1$, we conclude that there is statistical evidence to support a multiplicity of gust structures: If $\ell=1$, we conclude that there is no such evidence and that the stochastic process represented by the data is more likely Gaussian. 

The object of this section is to present a technique for standardizing the choice of $q$.

We first note that $q$ acts like a kind of scaling parameter. In effect, the larger the value of $q$, the greater the information about the remote past contained in a given $\phi_{k}$. We illustrate this point by computing a norm for $\psi_{k}$ defined by

$$
\left\|\psi_{k}\right\| \equiv \sqrt{\sum_{i=0}^{\infty} i\left|\psi_{k}(i)\right|^{2} q^{i}},
$$

a kind of mean for the distribution of $\psi_{k}$.

Theorem El:

$$
\| \psi_{k} !=\sqrt{\frac{a_{k, k-1}}{a_{k k}}-\frac{a_{k+1, k}}{a_{k+1, k+1}}}
$$

for $k=2,3, \ldots$.

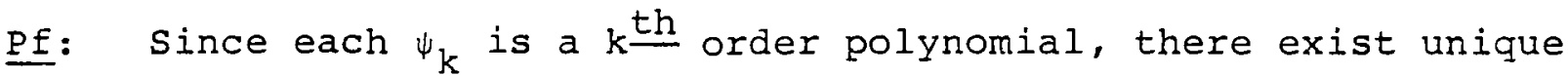
values for $c_{1}, \ldots, c_{k+1}$ such that

$$
i \psi_{k}(i)=\sum_{j=1}^{j+1} c_{j} \psi_{j}(i)
$$


By virtue of the orthonormality of the set $\left\{\psi_{k}\right\}_{k=1}^{\infty}$, we have

$$
\left\|\psi_{k}\right\|^{2}=\left(\sum_{j=1}^{k+1} c_{j} \psi_{j}^{\prime} \psi_{k}\right)=c_{k} .
$$

We proceed to compute $c_{k}$.

$$
\psi_{j}(i)=\sum_{m=1}^{j} a_{j m} i^{m-1}
$$

implies that

$$
\begin{aligned}
& \sum_{m=1}^{k} a_{k m} i^{m} \\
= & \sum_{j=1}^{k+1} c_{j} \sum_{m=1}^{j} a_{j m} i^{m-1} \\
= & \sum_{m=1}^{k+1}\left(\sum_{j=m}^{k+1} c_{j} a_{j m}\right)^{m-1}
\end{aligned}
$$

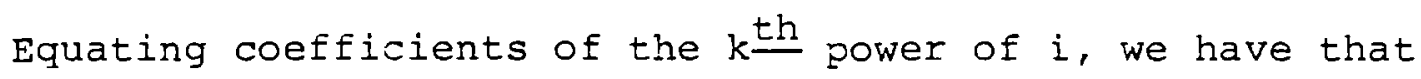

$$
c_{k+1}=\frac{a_{k k}}{a_{k+1, k+1}}
$$

Equating coefficients of the $(k-1)$ st power of $i$, we have that

$$
a_{k, k-1}=c_{k} a_{k k}+c_{k+1} a_{k+1, k}
$$


Thus,

$$
c_{k}=\frac{a_{k, k-1}}{a_{k k}}-\frac{a_{k+1, k}}{a_{k+1, k+1}}
$$

In addition, $\psi_{1}(i) \equiv \sqrt{1-q}$ implies that

$$
\begin{aligned}
& \left\|\psi_{1}\right\| \\
= & \sqrt{(1-q) \sum_{i=0}^{\infty} i q^{i}} \\
= & \sqrt{(1-q) q \frac{d}{d q}\left(\frac{1}{1-q}\right)} \\
= & \sqrt{\frac{q}{1-q}}
\end{aligned}
$$

Plots of $\left\|\psi_{k}\right\|$ vs. $q$ for $k=1, \ldots, 10$ are given in Figure $E 1$. The monotonicity of $\left\|\psi_{k}\right\|$ for each $k$ supports the claim that the information from the remote past represented by $\phi_{k}$ is an increasing function of $q$.

$$
\begin{aligned}
& \text { Now define the function } \sigma \text { by } \\
& \qquad \begin{aligned}
\sigma(t) & \equiv \max _{i, j} \max _{-l \leq k}\left|\rho_{i j}(k)\right|
\end{aligned}
\end{aligned}
$$

for $t$ in $[0, \infty)$, where $\left(p_{i j}\right)$ is the covariance matrix defined in Section II. $\sigma$ is essentially the smallest decreasing function on $[0, \infty)$ which dominates the elements of $\left(p_{i j}\right)$. We proceed to 


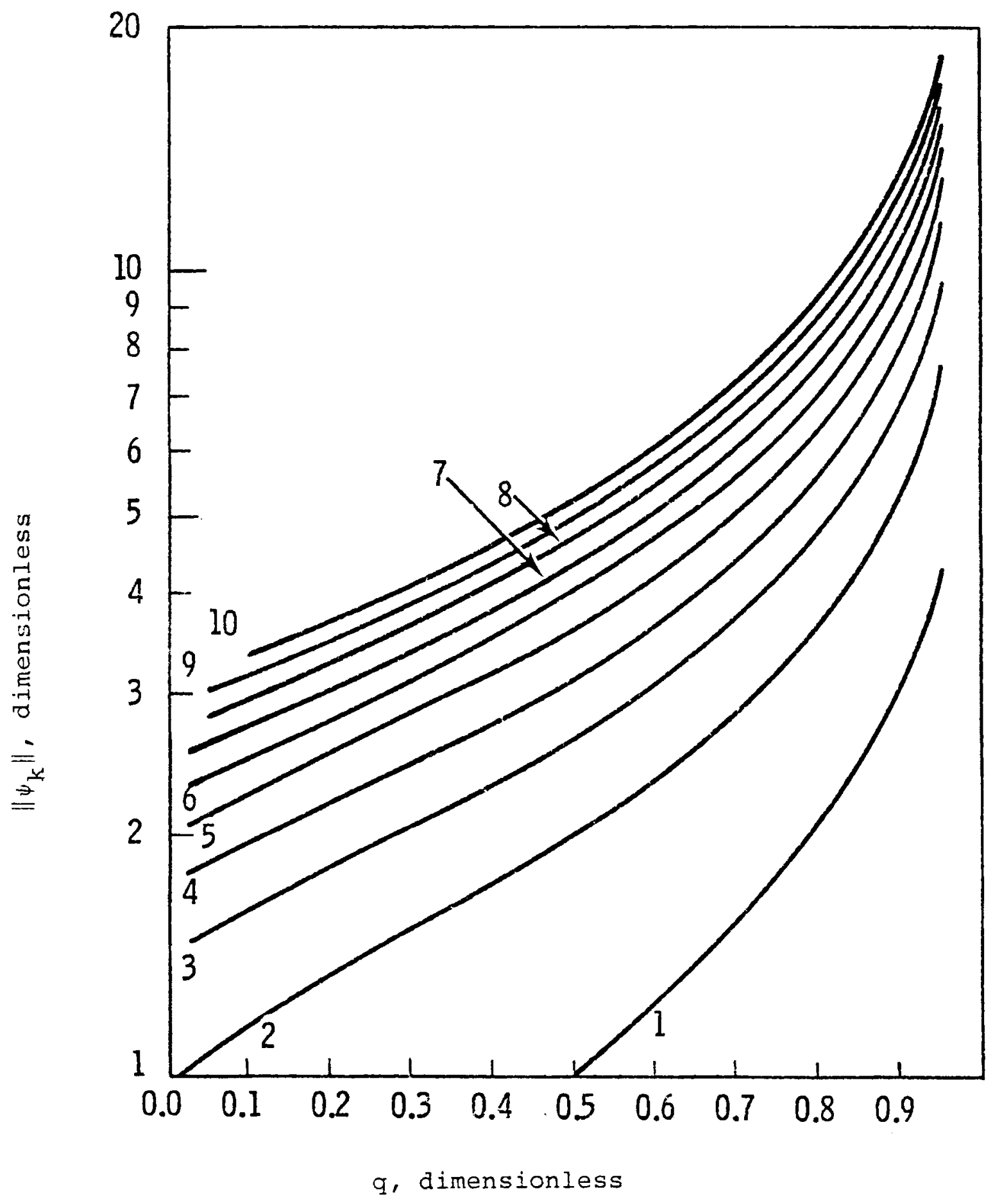

FIGURE El. Plots of $\left\|_{k}\right\|$ vs. q for various values 
use $\sigma$ to define a norm for $\left(\rho_{i j}\right)$. First, assuming square integrability, we fit $\sigma$ in some least squares sense by a function of the form

$$
\sigma(0) e^{-\alpha t^{\beta}}
$$

Next, consider

Theorem E2:

$$
\int_{0}^{\infty} t e^{-\alpha t^{\beta}} d t=\frac{\Gamma(2 / \beta)}{\beta \alpha}
$$

pf: Set $u \equiv \alpha t^{\beta}$. Then $t=\left(\frac{u}{\alpha}\right)^{1 / \beta}$ and $\frac{d t}{t}=\frac{1}{\beta} \frac{d u}{u}$. Thus,

$$
\begin{aligned}
& \int_{0}^{\infty} t e^{-\alpha t^{\beta}} d t \\
= & \int_{0}^{\infty}\left(\frac{u}{\alpha}\right)^{2 / \beta} e^{-u} \frac{1}{\beta} \frac{d u}{u} \\
= & \frac{1}{\beta \alpha^{2 / \beta}} \int_{0}^{\infty} e^{-u} u(2 / \beta)-1 d u \\
= & \frac{\Gamma(2 / \beta)}{\beta \alpha^{2 / \beta}} .
\end{aligned}
$$

Finally, define

$$
\left\|\left(o_{i j}\right)\right\| \equiv \sqrt{\int_{0}^{\infty} t \dot{\sigma}(0) e^{-\alpha t^{\beta}} d t},
$$


where $\alpha$ and $\beta$ are the parameters derived from the least squares fit. Then, by Theorem E2,

$$
\left\|\left(\rho_{i j}\right)\right\|=\sqrt{\frac{\sigma(0) \Gamma(2 / \beta)}{\beta \alpha^{2 / \beta}}} .
$$

$\left\|\left(\rho_{i j}\right)\right\|$ of course is also a kind of mean for the aistribution of the function $\max _{i, j}\left|\rho_{i, j}\right|$.

Finally, we stardardize the choice of $q$ by requiring it to satisfy $\left\|\psi_{N}\right\|=\left\|\left(\rho_{i j}\right)\right\|$. Figure $E l$ may be helpful in obtaining specific values. Other techniques for choosing $q$ are certainly possible. This one, however, places particular emphasis on the period of history in each sample path which is statistically most important in constructing $\mathrm{P}_{\mathrm{N}}$. 


\section{APPENDIX F \\ AN UPPER BOUND ON A SPECIAL CLASS \\ OF CROSSCOVARIANCE FUNCTIONS}




\section{AFPENDIX F \\ AN UPPER BOUND ON A SPECIAL CLASS \\ OF CROSSCOVARIANCE FUNCTIONS}

Let $g$ and $h$ denote two discrete stationary stochastic processes. Define the means $\bar{g}$ and $\bar{h}$ by

$$
\bar{g} \equiv E g(i), \bar{h} \equiv E h(i),
$$

and the crosscovariance function $\sigma$ by

$$
\sigma(j) \equiv E(g(i+j)-\bar{g})(h(i)-\bar{h}) .
$$

Define functions $\xi_{\mathrm{k}}$ and $\zeta_{\mathrm{k}}$ by

$$
\xi_{k}(j) \equiv \sum_{i=0}^{\infty} g(j-i) \psi_{k}(i) q^{i},
$$

and

$$
\zeta_{k}(j) \equiv \sum_{i=0}^{\infty} h(j-i) \psi_{k}(i) q^{i},
$$

for $k=1,2, \ldots$. Further, define the means $\bar{\xi}_{k}$ and $\bar{\zeta}_{k}$ by

$$
\bar{\xi}_{k} \equiv E \xi_{k}(i), \quad \bar{\zeta}_{k} \equiv E \zeta_{k}(i),
$$

for $k=1,2, \ldots$, and the crosscovariance functions $\sigma_{\ell m}$ by 


$$
\sigma_{2 m}(j) \equiv E\left(\xi_{l}(i+j)-\bar{\xi}_{2}\right)\left(\xi_{m}(i)-\bar{\zeta}_{m}\right)
$$

for $\ell, m=1,2, \ldots$. The object of this section is to compute an upper bound on $\left|\sigma_{\ell \mathfrak{m}}(k)\right|$ in terms of $\sigma, \psi_{\ell}, \psi_{\mathrm{m}}$ and $k$. Thus, by identifying $f_{i}$ with $g$, $f_{j}$ with $h_{1} \phi_{\ell i}$ with $\xi_{\ell}$, and $\phi_{m j}$ with $\zeta_{m}$ ' we have a way of estimating the values of $k$ for which $\phi_{\ell}(k+n)$ and $\phi_{m j}(n)$ are essentially independent.

We first turn attention to two technical lemmas.

Lemma Fl: $\quad\left|\psi_{k}(j)\right| \leq c_{k}\left(j^{k-1}+1\right)$ for $k=1,2, \ldots$, where $c_{k}=\sum_{i=1}^{k}\left|a_{k j}\right|$. Pf:

$$
\left|\psi_{k}(j)\right| \leq \sum_{i=1}^{k}\left|a_{k i}\right| j^{i-1} \leq\left(\sum_{i=1}^{k}\left|a_{k i}\right|\right)\left(j^{k-1}+1\right)
$$

\section{Lemma F2:}

$$
\left|\sum_{i=0}^{\infty} \psi_{\ell}(i) \psi_{m}(i+j) q^{2 i}\right|<c_{\ell} c_{m} s(l+m-2) q^{-2}(j+1)^{m-1}
$$

Pf: First, Lemma Fl implies that

$$
\begin{aligned}
& \left|\sum_{i=0}^{\infty} \psi_{\ell}(i) \dot{\psi}_{m}(i+j) q^{2 i}\right| \\
& \leq c_{\ell} c_{m} \sum_{i=0}^{\infty}\left(i^{\ell-1}+1\right)\left((i+j)^{m-1}+1\right) q^{2 i} .
\end{aligned}
$$

Next, a simple induction shows that 


$$
(i+j)^{n}+1 \leq(i+1)^{n}(j+1)^{n}
$$

for $i, j \geq 0$ and $n=1,2, \ldots$ :

$$
\begin{aligned}
& (i+j)^{n+1}+1 \\
= & \left((i+j)^{n}+1\right)(i+j)-(i+j)+1 \\
\leq & (i+1)^{n}(j+1)^{n}(i+j)+1 \\
\leq & (i+1)^{n}(j+1)^{n}(i+j+1) \\
\leq & (i+1)^{n+1}(j+1)^{n+1} .
\end{aligned}
$$

Thus,

$$
\left(i^{\ell-1}+1\right)\left((i+j)^{m-1}+1\right) \leq(j+1)^{m-1}(i+1)^{\ell+m-2}
$$

Therefore,

$$
\begin{aligned}
& \mid \sum_{i=0}^{\infty} \psi_{\ell}(i) \psi_{m}(i+j) q^{2 i} \\
\leq & c_{\ell} c_{m} q^{-2}(j+1)^{m-1} \sum_{i=0}^{\infty}(i+1)^{\ell+m-2} q^{2(i+1)} \\
< & c_{\ell} c_{m} q^{-2}(j+1)^{m-1} \sum_{i=0}^{\infty} i^{\ell+m-2} q^{i} .
\end{aligned}
$$


Theorem Fl: $\quad\left|\sigma_{\ell m}(k)\right| \leq|\sigma| * k_{\hat{x}, \mathrm{M}}(k)$,

where * denotes convolution and

$$
\mathrm{K}_{\ell \mathrm{m}}(k) \equiv \mathrm{C}_{\ell} \mathrm{C}_{\mathrm{m}} \mathrm{S}(\ell+\mathrm{m}-2) \mathrm{q}|\mathrm{k}|-2(|\mathrm{k}|+1)^{\ell \sim \mathrm{m}-1} .
$$

Pf: $\left|\sigma_{\ell m}(k)\right|$

$$
\begin{aligned}
& =E\left[\left(\xi_{\ell}(k+n)-\bar{\xi}_{\ell}\right)\left(\zeta_{m}(n)-\bar{\zeta}_{m}\right)\right] \\
& =E\left[\left(\sum_{i=0}^{\infty} g(k+n-i) \psi_{\ell}(i) q^{i}-E \sum_{i=0}^{\infty} g(k+n-i) \psi_{\ell}(i) q^{i}\right)\right. \\
& =\sum_{i=0}^{\infty} \sum_{j=0}^{\infty} E[(g(k+n-i)-\bar{g})(n(n-j)-\bar{n})] \psi_{\ell}(i) \psi_{m}(j) q^{i+j} \\
& \left.\left.=\sum_{i=0}^{\infty} \sum_{j=0}^{\infty} \sigma(k-(i-j)) \psi_{\ell}(i) \psi_{m}(j) q^{i+j}(j) q^{j}-E \sum_{j=0}^{\infty} h(n-j) \psi_{m}(j) q^{j}\right)\right] \\
& =\sum_{i=0}^{\infty} \sum_{j=i}^{\infty} \sigma\left(k+(j-i) \psi_{\ell}(i) \psi_{m}((j-i)+i) q^{2 i} q^{j-i}\right. \\
& +\sum_{j=0}^{\infty} \sum_{i>j} \sigma(k-(i-j)) \psi_{\ell}((i-j)+j) \psi_{m}(j) q^{i-j} q^{2 j} \\
& +\sum_{i=0}^{\infty} \sum_{j=0}^{\infty} \sigma(k+j) \psi_{\ell}(i) \psi_{m}(i+j) q^{2 i} q^{j} \\
& +\sum_{j=0}^{\infty} \sum_{i=1}^{\infty} \sigma(k-i) \psi_{\ell}(i+j) \psi_{m}(j) q^{i} q^{2 j} .
\end{aligned}
$$


A general discussion about interchanging the operations $\sum$ and $E$ is given in [9].

Thus,

$$
\begin{aligned}
& \left|\sigma_{\ell m}(k)\right| \\
\leq & \sum_{j=0}^{\infty}|\sigma(k+j)| q^{j}\left|\sum_{i=0}^{\infty} \psi_{\ell}(i) \psi_{m}(i+j) q^{2 i}\right| \\
+ & \sum_{j=1}^{\infty}|\sigma(k-j)| q^{j}\left|\sum_{i=0}^{\infty} \psi_{\ell}(i+j) \psi_{m}(i) q^{2 i}\right| \\
\leq & \sum_{j=0}^{\infty}|\sigma(k+j)| q^{j} c_{\ell} c_{m} s(\ell+m-2) q^{-2}(j+1)^{m-1} \\
+ & \sum_{j=1}^{\infty}|\sigma(k-j)| q^{j} c_{\ell} c_{m} s(\ell+m-2) q^{-2}(j+1)^{\ell-1} \\
= & \sum_{j=-\infty}^{\infty}|\sigma(k-j)| c_{\ell} c_{m} s(\ell+m-2) q|j|-2(|j|+1)^{\ell / m-1} .
\end{aligned}
$$


No. of Copies

OFFSITE

27 DOE Technical Information Center

10 T. C. Kerrigan

Mathematics Department

Drexel University

Philadelphia, PA 19104

ONSITE

2 DOE Richland Operations Office

G. L. Liffick

H. E. Ransom

30 Pacific Northwest Laboratory

W. C. Cliff

J. R. Connell

R. L. Conley

J. C. Doran

R. I. Drake

C. E. Elderkin (5)

D. L. EIIiott

M. M. Orgil1

P. R. Partch

W. T. Pennell

E. H. Phinney

J. V. Ramsdel1

D. S. Renne

C. L. Simpson

L. L. Wendell (5)

Technical Information (5)

Publishing Coordination (2) 